\title{
A BI-FUNCTIONAL POLYMERIC COATING FOR THE CO-IMMOBILIZATION OF PROTEINS AND PEPTIDES ON MICROARRAY SUBSTRATES
}

\author{
Laura Sola*, Dario Brambilla, Alessandro Mussida, Roberto Consonni, Francesco Damin, Marina Cretich, \\ Alessandro Gori and Marcella Chiari \\ Istituto di Scienze e Tecnologie Chimiche "G.Natta" \\ National Research Council of Italy \\ Via Mario Bianco 9 \\ 20131 Milan Italy
}

* To whom correspondence should be addressed:

Dr. Laura Sola

e-mail: laura.sola@scitec.cnr.it

telephone: +3902 28500042

Keywords: Click chemistry; functional polymers; co-immobilization; protein microarray, peptide microarray; extracellular vesicles. 


\section{Abstract}

The analytical performance of the microarray technique in screening the affinity and reactivity of several probes towards a specific target, is highly affected by the coupling chemistry adopted to bind probes to the surface. However, the surface functionality limits the biomolecules that can be attached to the surface to a single type of molecule (DNA, protein, or peptide), thus forcing the execution of separate analyses to compare the performance of different species in recognizing their targets. Here we introduce a new $N, N$-dimethylacrylamide-based polymeric coating, bearing simultaneously different functionalities ( $N$-Acryloyloxysuccinimide and azide groups) to allow an easy and straightforward method to co-immobilize proteins and oriented peptides on the same substrate. The bi-functional copolymer has been obtained by partial post polymerization modification of the functional groups (NAS) of a common precursor. A deep characterization of the copolymer was carried out by means of NMR to quantify the percentage of NAS that has been transformed into azido groups. The polymer was then used to coat surfaces onto which both native antibodies and alkyne modified peptides were immobilized, to perform the phenotype characterization of extracellular vesicles (EVs). Ultimately, this strategy represents a convenient method to reduce the number of analysis, thus possible systematic or random errors, besides offering a drastic shortage in time, reagents and costs.

\section{Introduction}

In the last decades microarrays-based analytical devices have gained increasing importance in clinical field and in the development of biosensors, thanks to their high throughput and multiplexing capability. In fact, the literature reports hundreds of applications in biomedical analysis, based on this technology [1][2][3]. Their operating principle relies on the specific interaction between biomolecules, namely probes, immobilized on the surface and analyte molecules in solution. In particular, a single class of molecules is immobilized on a microarray surface, consequently they are generally classified as DNA, protein, peptide, glycan or cell microarrays. The reason why only a single type of molecule is bond to the support mainly depends on the chemistry adopted to link the probes to the surface, which must confer stability but also maintain the molecule reactivity. The most common and exploited strategy, in fact, consists in forming an organic layer bearing functional groups that can establish interactions with the probe by means of physical forces or chemical bonding. As a matter of fact, only a single type of functionality is used to the generation of a 
homogeneous coating, thus limiting the molecules that can be simultaneously attached to the surface. However, in some occasions, there might be the need to co-immobilize probes that do not share the same functionality. For example, Gori and collaborators have recently demonstrated that the accurate control of peptidic probe immobilization, obtained by a click chemistry strategy, can greatly affect the analytical outcome of microarrays for serological applications. However, the comparison between the behavior of the linear peptidic epitopes versus that of the full protein antigens from which they are derived, could not have been performed on the same slide because their immobilization strategies are incompatible [4].

Literature reports few examples of co-immobilization of different biomolecules, which are usually bound to the surface exploiting a single-type chemistry [5]. Multi-chemistry arrays have been introduced for the high-throughput screening of new materials for cell cultures [6][7]. In this approach, pre-synthesized polymers were arrayed on a support, and then different cell lines were cultured on top of each spot, which represents a different biomaterial. Recently, Rigo and coworkers have introduced a dually functionalized surface, based on SPAAC and thiol-ene click reactions, to simultaneously immobilize two nanoassemblies to obtain controlled patterns on the surface[8]. Similarly, Deng and collaborators, proposed a co-immobilization method, based on click chemistry, for heparin-binding growth factor and adhesion peptide on the copolymer obtained by chemical vapor deposition (CVD) with the co-presence of aldehyde and alkyne groups. Heparinbinding growth factor was then immobilized through interaction with heparin, which was covalently attached to the surface through an aldehyde-hydrazide reaction. In parallel, a classic alkyne-azide click reaction was used to orthogonally co-immobilize an adhesion peptide as the second biomolecule [9].

Here we propose a new polymeric coating bearing different functionalities, allowing an easy and straightforward method to co-immobilize biomolecules on the same slide through different functional groups. We have recently introduced a family of $\mathrm{N}, \mathrm{N}$-dimethylacrylamide (DMA)-based copolymers that form a thin film on different materials by a combination of physi- and chemisorption [10]. Starting from a common precursor that bears $N$-Acryloyloxysuccinimide (NAS) as functional group (highly reactive toward nucleophiles such as the amines present in proteins and peptides), by simple post polymerization modifications, a family of copolymers bearing groups such as azide, alkyne, DBCO and thiols was introduced. The presence of these moieties allows probe immobilization by means of click chemistry reactions. In this work, instead of modifying entirely the functional groups of the parent polymer, only a specific percentage was modified, in order to obtain 
a copolymer that bears simultaneously two distinct functionalities, in particular NAS and azide. A deep characterization of the copolymers was carried out by means of NMR to quantify the percentage of NAS that has been modified. The polymer was then used to coat surfaces onto which both native antibodies and alkyne modified peptides have been simultaneously immobilized. This strategy allows the phenotype characterization of extracellular vesicles (EVs).

\section{Materials and Methods}

N,N-Dimethylacrylamide (DMA), 3-(trimethoxylsilyl)propyl methacrylate (MAPS), copper sulfate (CuSO4), ascorbic acid, Tris(3-hydroxypropyltriazolylmethyl)amine (THPTA), $\alpha, \alpha^{\prime}-$ Azoisobutyronitrile (AIBN), anhydrous tetrahydrofuran (THF), ammonium sulphate $\left(\left(\mathrm{NH}_{4}\right)_{2} \mathrm{SO}_{4}\right)$, phosphate buffered saline (PBS), ethanolamine, concentrated ammonia $\left(\mathrm{NH}_{3}\right)$, 3(diethylamino)propylamine and Rabbit IgG were purchased from Sigma Aldrich (St. Louis, MO, USA). All solvents were used as received. $\mathrm{N}$-acryloyloxysuccinimide and 3-azido-1-propylamine were synthesized as reported elsewhere[11] [12]. All the oligonucleotides for hybridization tests, were synthesized by MWG-Biotech AG (Ebevsberg, Germany) with the following sequences: 5'GCCCACCTATAAGGTAAAAGTGA-3' (COCU8; modified in 5' with the following functionalities: amine (NH2), dibenzocyclooctyne (DBCO), azide (N3), 5'-Cy3-TCACTTTTACCTTATAGGTGGGC-3' (COCU10), COCU10 was labeled with the fluorophore Cyanine 3 for fluorescence detection. These oligonucleotides were freeze-dried and re-suspended in DI water at a final concentration of $100 \mu \mathrm{M}$ before use.

Peptide E01, modified with alkyne group, has the following sequence: $\operatorname{Prg}-\left(\mathrm{O}_{2} \mathrm{Oc}\right)_{2^{-}}$ DRGWGNGCGLFG and has been synthesized as reported in [13]. BP( RPPGFSPFRKG- $\left(\mathrm{O}_{2} \mathrm{O}_{2}\right)-\operatorname{Prg}$ and BPn ( EPPGFSPFEKG- $\left(\mathrm{O}_{2} \mathrm{O}_{2}\right)$-Prg) peptides have been synthesized as reported in [14]. Rabbit anti-E01 antibody is a kind gift from Primm srl (Milano, Italy). For rabbit anti-E01 detection, Cy-3 labelled goat anti-rabbit IgG was obtained from Jackson Immunoresearch (West Grove, PA, USA). Mouse anti-human CD9 IgG (clone MEM-61) and mouse anti-human CD63 IgG (clone MEM-259) were provided by Hansa BioMed Life Sciences Ltd (Tallinn, Estonia). Cy3-labelled anti-human CD81 IgG was part of ExoView Tetraspanin Plasma kit (NanoView Biosciences Inc., MA, USA).

Untreated silicon slides $1000 \AA ̊$ Thermal Oxide (14 X $14 \mathrm{~mm}$ ) were supplied by SVM, Silicon Valley Microelectronics Inc. (Santa Clara, CA USA). NV10B silicon chips were supplied by NanoView Biosciences (Boston, MA, USA). Both chips were pretreated using a HARRICK Plasma Cleaner, PDC- 
002 (Ithaca, NY, USA) connected to an oxygen line. Spotting is performed using a SciFLEXARRAYER S12 (Scienion, Berlin, Germany).

Fluorescence images were obtained using the ScanArray Lite confocal laser scanner and analyzed using ScanArray Express software (Perkin Elmer, MA, USA). Interferometric analysis of EVs was performed using ExoView ${ }^{\mathrm{TM}}$ R100 for image acquisition and NanoViewer 2.6.0 software for analysis (NanoView Biosciences Inc., MA, USA). Nanoparticle Tracking Analysis was performed with NanoSight NS300 using 3.2 Dev Build 3.2.16 software (Malvern Instruments Ltd, Malvern, United Kingdom).

\subsection{Polymer synthesis}

All copolymers have been obtained by post-polymerization modification reactions of the parent polymer MCP4 which is composed of DMA ( $89 \%$ molar fraction), NAS (10\% molar fraction), and 3(trimethoxysilyl)- propyl methacrylate (MAPS, 1\% molar fraction) (see Figure 1). The parent polymer MCP4 was synthesized by free radical polymerization according to the procedure described in [15]. Briefly, after degassing anhydrous THF with argon, DMA, NAS, and MAPS were added to the reaction flask so that the total monomer feed was $20 \% \mathrm{w} / \mathrm{v}$. The reaction mixture was heated to $65^{\circ} \mathrm{C}$ for two hours in the presence of AIBN. The crude material was cooled to room temperature and diluted 1:1 with dry THF; the solution was then precipitated in petroleum ether (10 times the volume of the reaction mixture) to eliminate unreacted monomers. The polymer was collected by filtration as a white powder and dried under vacuum at room temperature overnight. To introduce azide moiety a $10 \% \mathrm{w} / \mathrm{v}$ solution of the copolymer was prepared by dissolving it in dry THF; the mixture was purged with argon and then azido-1-propylamine was added with a 2.5 molar ratio for Copoly azide 10\%, 0.2 molar ratio for Copoly Mix 1, and 0.5 molar ratio for Copoly Mix 2 with respect to the moles of NAS which concentration along the polymer chain is $100 \mathrm{mM}$ (see Figure 1). The mixture was stirred for $5 \mathrm{~h}$ at room temperature and then the polymers were precipitated in petroleum ether (10 times the volume of the reaction mixture), filtered on a Büchner funnel, and dried under vacuum at room temperature. 


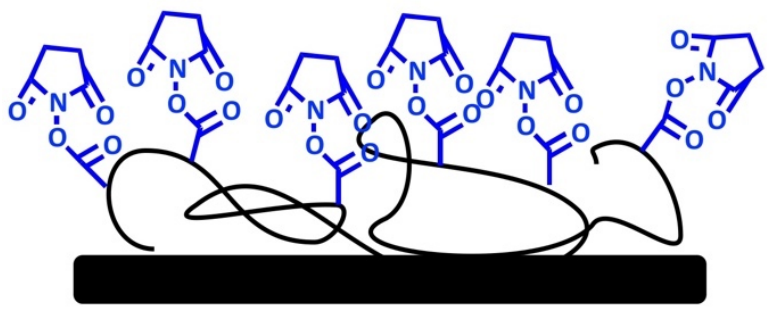

MCP4

NAS molar ratio: $10 \%$

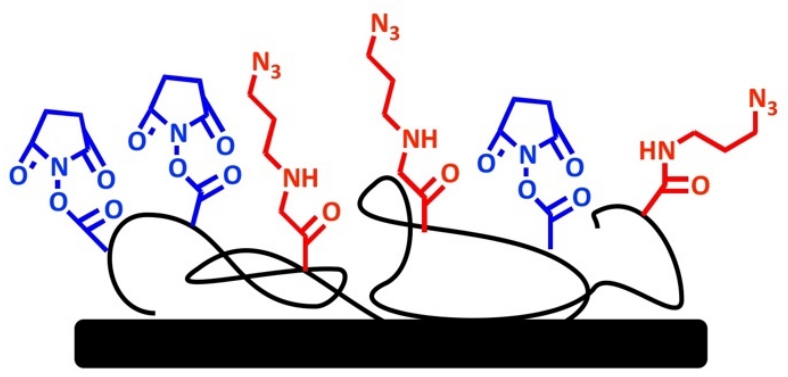

Copoly Mix 2

NAS molar ratio: $5 \%$

Azide molar ratio: $5 \%$

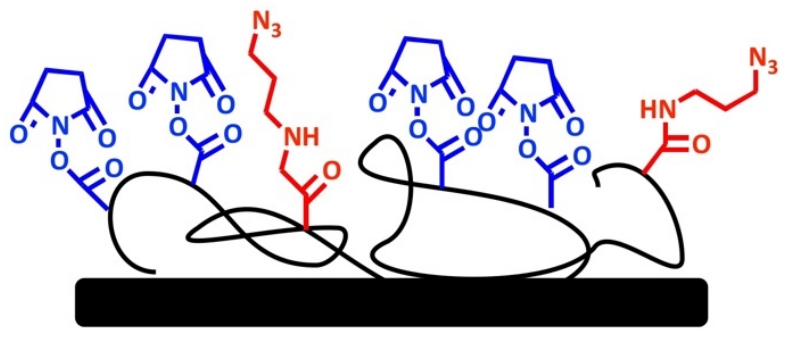

Copoly Mix 1

NAS molar ratio: $8 \%$

Azide molar ratio: $2 \%$

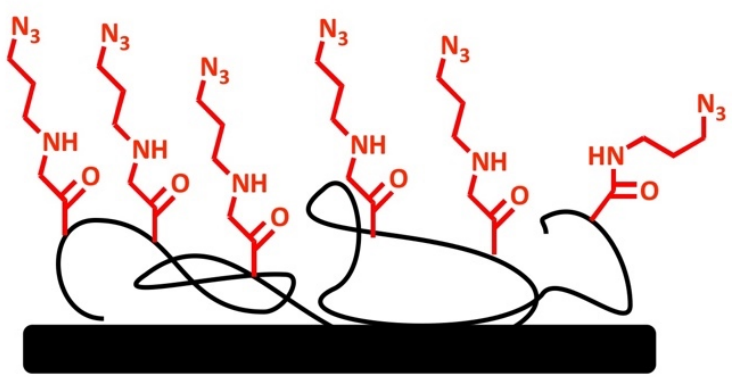

Copoly Azide 10\%

Azide molar ratio: $10 \%$

Figure 1 Schematic representation of the parent polymer MCP4 and modified polymers with azide groups. MCP4 is a copolymer composed of DMA, MAPS and NAS as functional groups, whose molar ratio is $10 \%$ of the total monomer feed. By modifying $2 \%$ of the NAS functionalities with azidopropylamine, Copoly Mix 1 is obtained; similarly Copoly Mix 2 is obtained by transforming half of the NAS groups into azido moieties. A quantitative MCP4 post polymerization modification yields to Copoly Azide 10\%.

\subsection{NMR Characterization}

MCP4, Copoly Mix 2 and Copoly Azide 10\% polymers have been characterized by acquiring ${ }^{1} \mathrm{H}$ and ${ }^{13} \mathrm{C}$ NMR spectra at $500 \mathrm{MHz}$ Bruker Avance II and $600 \mathrm{MHz}$ Bruker Avance DRX spectrometers equipped with reverse $5 \mathrm{~mm}$ TXI probe with z gradient. About $30 \mathrm{mg}$ of polymer were dissolved in DMSO- $d^{6}$ solvent. All spectra were recorded at $300 \mathrm{~K}$ and calibrated on solvent signal resonating at $2.5 \mathrm{ppm}\left({ }^{1} \mathrm{H}\right)$ and $40.5 \mathrm{ppm}\left({ }^{13} \mathrm{C}\right)$. Monodimensional ${ }^{13} \mathrm{C}$ spectra have been acquired with the inverse gated decoupling sequence to avoid heteronuclear Overhauser effect, with sufficient relaxation delay to allow the complete magnetization recovery among pulses. Spectral width of $37800 \mathrm{~Hz}$ have been acquired over $64 \mathrm{~K}$ points for carbon, while $8012 \mathrm{~Hz}$ were acquired over $32 \mathrm{~K}$ point for proton spectra. Heteronuclear experiments (HSQC, HMBC) were also acquired for resonance assignment. 


\subsection{Surface coating}

Silicon oxide slides were pretreated with oxygen plasma for $10 \mathrm{~min}$ : the oxygen pressure was set to 1.2 bar with a power of 29.6 W. Each copolymer was dissolved in DI water to a final concentration of $2 \% \mathrm{w} / \mathrm{v}$ and then diluted 1:1 with a solution of ammonium sulfate $1.6 \mathrm{M}$. The slides were immersed into each solution for $30 \mathrm{~min}$, then rinsed with DI water, dried under a nitrogen stream, and finally cured under vacuum at $80^{\circ} \mathrm{C}$ for $15 \mathrm{~min}$.

\subsection{Surface functional test}

2.3.1 Spotting Silicon slides were coated as reported in Section 2.3. In particular 4 silicon chips were coated with MCP4, Copoly Azide 10\%, Copoly Mix 2 and Copoly Mix 1. The oligonucleotide 5'GCCCACCTATAAGGTAAAAGTGA-3' (COCU8), modified with $\mathrm{NH}_{2}$ or DBCO group was dissolved in printing buffer ( $150 \mathrm{mM}$ sodium phosphate buffer solution at $\mathrm{pH} 8.5$ containing $0.01 \%$ of sucrose monolaurate) to a final concentration of $1,10,25$ and $50 \mu \mathrm{M}$. The solutions of COCU8 $\mathrm{NH}_{2}$ and COCU8 DBCO were deposited onto the surface of the same chip using a noncontact microarray spotter (Scienion sciFLEXARRAYER S12) using a $80 \mu \mathrm{m}$ nozzle (see scheme in Figure 2). The spot volume, temperature, and humidity were precisely controlled to $400 \mathrm{pL}, 22{ }^{\circ} \mathrm{C}$, and $65 \%$, respectively. Immediately after spotting, all chips were stored overnight in a sealed chamber, saturated with sodium chloride $\left(40 \mathrm{~g} / 100 \mathrm{~mL} \mathrm{H}{ }_{2} \mathrm{O}\right)$.

After incubation, the silicon chips coated with MCP4, Copoly Mix 2 and Copoly Mix 1 were treated with an ethanolamine blocking solution (50 mM in $0.1 \mathrm{M} \mathrm{Tris} / \mathrm{HCl}$ buffer $\mathrm{pH}$ 9), preheated, and kept at $50{ }^{\circ} \mathrm{C}$ for $15 \mathrm{~min}$. The slide coated with Copoly Azide $10 \%$ did not need this step. Consequently, all chips were rinsed with DI water and immersed in a solution containing 4× SSC (600 mM sodium chloride, $60 \mathrm{mM}$ sodium citrate, and $\mathrm{pH} 7.0$ ) and $0.1 \%$ SDS, preheated at $50{ }^{\circ} \mathrm{C}$, and kept at this temperature for $15 \mathrm{~min}$, before rinsing with DI water and drying under a nitrogen stream.

2.3.2 Hybridization The printed chips were incubated with a complementary oligonucleotide target, 5'-Cy3-TCACTTTTACCTTATAGGTGGGC- 3' (COCU10) tagged with cyanine 3 for fluorescence detection. COCU10 was diluted to a final concentration of $1 \mu \mathrm{M}$ in an aqueous solution containing $2 \times \mathrm{SSC}, 0.1 \% \mathrm{SDS}$, and $0.2 \mathrm{mg} / \mathrm{mL}$ of $\mathrm{BSA} ; 15 \mu \mathrm{L}$ of this solution was layered on the hybridization area and covered with a coverslip. The reaction was performed in a humid chamber at $65{ }^{\circ} \mathrm{C}$ for 2 h. Finally, the chips were washed with a $4 \times$ SSC solution at room temperature (to remove the coverslip), and then any unbound oligonucleotide was removed using two successive rinses (5 min 
each) with a $2 \times \mathrm{SSC} / 0.1 \% \mathrm{SDS}$ solution, prewarmed at hybridization temperature $\left(65^{\circ} \mathrm{C}\right)$. Other two washes with $0.2 \times$ SSC and $0.1 \times$ SSC, carried out both at room temperature for $1 \mathrm{~min}$, were then performed, and finally, the slides were dried under a nitrogen stream. Fluorescence images of each chip were obtained using a confocal laser scanner (ScanArray Lite, Perkin Elmer), with the laser power set at $22 \%$ and the photomultiplier tube (PMT) gain at $64 \%$ and analyzed using the ScanArray Express software (see Figure 2).

a

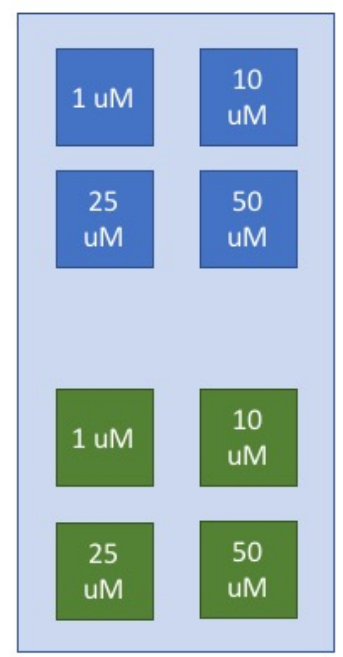

Cocu $8 \mathrm{NH}_{2}$ b

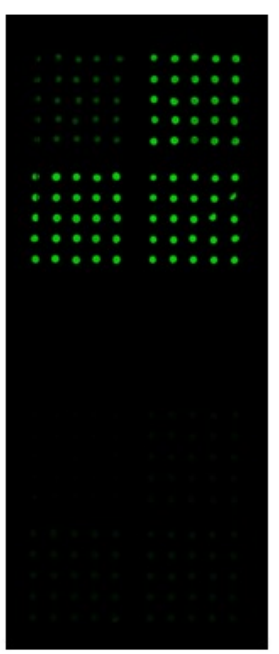

MCP4

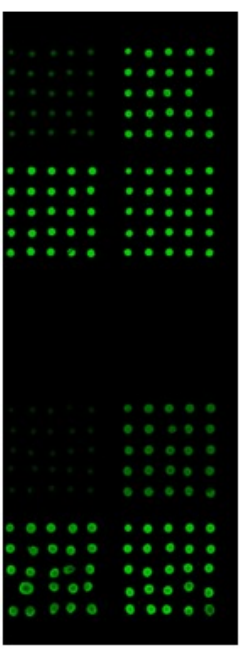

Copoly Mix 1

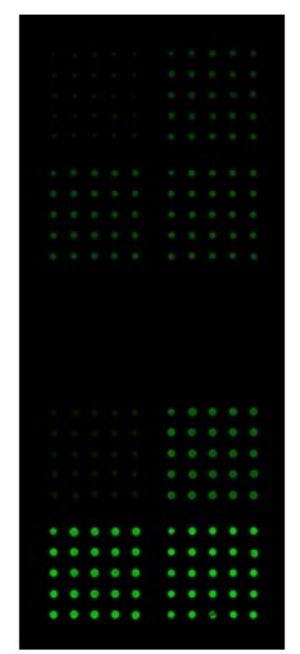

Copoly Mix 2

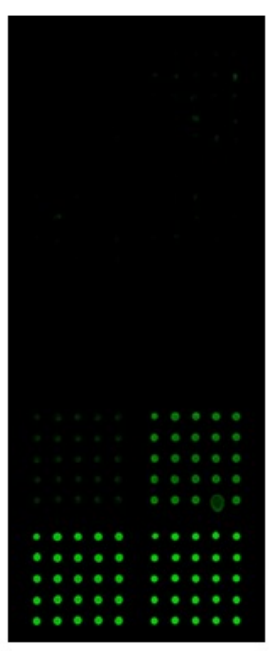

Copoly azide $10 \%$

\section{Cocu 8 DBCO}

Figure 2 a) Spotting scheme of the amino and DBCO modified oligonucleotide immobilized on MCP4, Copoly Mix 1, Copoly Mix 2 and Copoly Azide 10\% coated silicon slides; b) fluorescent images obtained after hybridization with the complementary DNA strand labelled with Cy3 for fluorescent detection. Slides were analyzed with a confocal laser scanner setting power at 22\% and PMT gain at $64 \%$.

\subsection{Protein and peptide co-immobilization}

On silicon chips coated with MCP4, Copoly Azide 10\%, Copoly Mix 2 and Copoly Mix 1 as reported in section 2.3 a solution of Rabbit $\operatorname{lgG} 1 \mathrm{mg} / \mathrm{mL}$ in PBS was immobilized using a Scienion sciFLEXARRAYER S12 spotter using a $80 \mu \mathrm{m}$ nozzle. The spot volume, temperature, and humidity were precisely controlled to $400 \mathrm{pL}, 22{ }^{\circ} \mathrm{C}$, and $65 \%$, respectively. Immediately after spotting, all chips were stored overnight in a sealed chamber, saturated with sodium chloride $\left(40 \mathrm{~g} / 100 \mathrm{~mL} \mathrm{H}_{2} \mathrm{O}\right)$. After incubation, all the chips (except for Copoly Azide 10\% which does not need this step) were treated with $50 \mathrm{mM}$ ammonia solution to block reactive site.

All the chips were then spotted again with solution of peptide E01 $100 \mu \mathrm{M}$ containing a click mix reagents composed of $\mathrm{CuSO}_{4} /$ ascorbic acid/THPTA $(6.525,0.1$, and $0.4 \mathrm{mM}$, respectively) to perform the click reaction. All the chips were then incubated again in a sealed chamber, saturated with 
sodium chloride, overnight. All the chips were then incubated for $1 \mathrm{~h}$ at room temperature with a 2 $\mathrm{mM}$ solution with EDTA, rinsed with water and finally dried with a nitrogen stream. As negative control, peptide E01 was spotted also without the click mix reagents.

All the chips were then incubated with a solution of rabbit IgG directed against E01 $0.1 \mathrm{mg} / \mathrm{ml}$ in PBS for 1 hour at room temperature in a humid chamber. Slides were then rinsed with washing buffer for 10 minutes (Tris/ $\mathrm{HCl}$ pH $90.05 \mathrm{M}, \mathrm{NaCl} 0.25 \mathrm{M}$, Tween 20 0.05\%), then with PBS for 10 minutes. Finally the slides were incubated with a solution of anti-rabbit IgG labelled with Cy3 for fluorescence detection $1 \mu \mathrm{g} / \mathrm{mL}$ in PBS for 1 hour at room temperature. Slides were washed with washing buffer for 10 minutes, then with PBS for 10 minutes and finally analyzed with a confocal laser scanner ScanArray Lite, Perkin Elmer), setting the laser power set at $60 \%$ and the photomultiplier tube (PMT) gain at 70\% and analyzed using the ScanArray Express software (Figure 3 a).

As negative control chips were also incubated directly with the Cy3 Anti-Rabbit IgG solution (see scheme in Figure $3 \mathrm{~b}$ ).

a

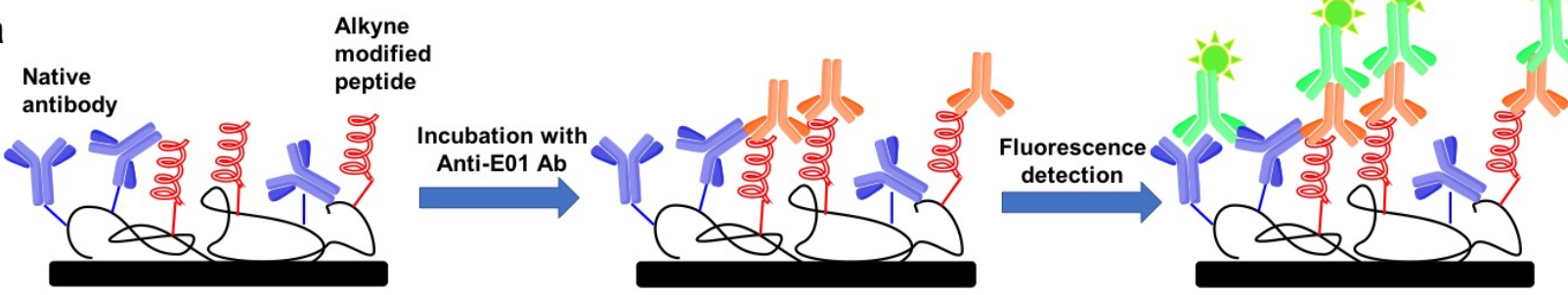

b

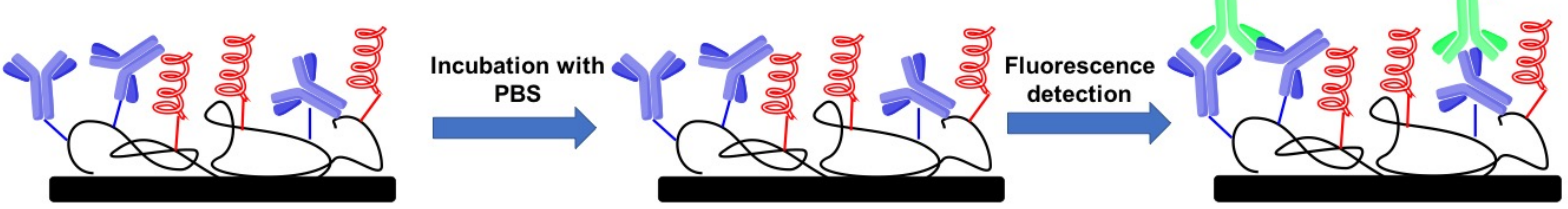

Figure 3 Schematic representation of the protein and peptide co-immobilization experiment. A native antibody and an alkyne modified peptide are immobilized on a Copoly Mix 2 coated silicon slide; a) the slide is incubated with an anti-EO1 antibody and then with a Cy3 labelled antibody for fluorescence detection; b) negative control: the slide is incubated with a blank sample not containing the antiE01 antibody and then with Cy3 labelled antibody to test the specificity of the assay.

\subsection{Separation and characterization of HEK-293-derived extracellular vesicles}

2.5.1 HEK-derived EVs separation by ultracentrifugation. Three days conditioned media from HEK293 cells were harvested and centrifuged at $500 \times \mathrm{g}$ for 25 minutes. Supernatants were filtered with $0.22 \mathrm{~mm}$ filters (Merck Millipore) and centrifuged in a SorvallTM WX Ultracentrifuge (ThermoFisher Scientific, WX Ultra $100 \# 75000100$ ) at $150.000 \times$ g for 90 minutes at $4^{\circ} \mathrm{C}$ with a SureSpinTM 630 swinging bucket rotor (ThermoFisher Scientific) to pellet EVs. After supernatant was carefully removed, EV-containing pellet were resuspended in PBS and directly used for subsequent analysis. 
2.5.2 HEK-derived EVs characterization using Nanoparticle Tracking Analysis. The sample obtained as reported in paragraph 2.5.1 was analyzed using Nanosight NS300 (Malvern Panalytical, Malvern, UK). Videos were analyzed by the in-built NanoSight Software NTA 3.2 Dev Build 3.2.16. The Camera type, Camera level, and Detect Threshold were sCMOS, 14 and 4, respectively. The number of completed tracks in NTA measurements was 5 (a 60 seconds movie was registered for each measurement). Sample was diluted in PBS to a final volume of $1 \mathrm{~mL}$. The ideal concentration was assessed by pre-testing the optimal particle per frame value (20-100 particles per frame).

\subsection{Analysis of extracellular vesicles using label-free interferometry}

2.6.1 Chip functionalization. SP-IRIS silicon chips were coated using Copoly Mix 2 described in Section $2.3 .1 \mathrm{mg} / \mathrm{mL}$ solutions in PBS of three antibodies (namely antiCD9, antiCD63 to capture EVs and rabbit IgG as negative control) were deposited using a Scienion sciFLEXARRAYER S12 spotter equipped with a $80 \mu \mathrm{m}$ nozzle. The spot volume, temperature, and humidity were precisely controlled to $400 \mathrm{pL}, 22^{\circ} \mathrm{C}$, and $65 \%$, respectively. Immediately after spotting, all chips were stored overnight in a sealed chamber, saturated with sodium chloride $\left(40 \mathrm{~g} / 100 \mathrm{~mL} \mathrm{H} \mathrm{H}_{2} \mathrm{O}\right)$. After incubation the chips were treated with $50 \mathrm{mM}$ 3-(diethylamino)propylamine solution to block reactive sites, rinsed with bidistilled water and dried. All the chips were then spotted again with $100 \mathrm{mM}$ solution of peptides (BP, a peptide that strongly binds small EVs, and BPn as negative control) dissolved in a click mix solution composed of $5 \mathrm{mM} \mathrm{Na}$ /Acetate $\mathrm{pH}$ 4.8, $50 \mathrm{mM}$ Trehalose, $100 \mu \mathrm{M}$ CuSO4, $400 \mu \mathrm{M}$ THPTA and $6.25 \mathrm{mM}$ Ascorbic Acid to perform the CuAAC reaction. The chips were then incubated again in a sealed chamber, saturated with sodium chloride, overnight. Then, the chips were incubated for $1 \mathrm{~h}$ at room temperature with $2 \mathrm{mM}$ EDTA, rinsed with bidistilled water and finally dried.

2.6.2 Capture and detection of extracellular vesicles. Silicon chips, prepared as described in Section 2.6.1 were analyzed using ExoView R100 to obtain prescan images and then divided into 2 groups. One group was incubated with HEK-derived EVs $\left(2 * 10^{9}\right.$ particles/mL, diluted in PBS) for $2.5 \mathrm{~h}$ at room temperature. After incubation the chips were washed $10 \mathrm{~min}$ in PBS, rinsed with bidistilled water and dried. The second group was incubated with PBS (as negative control) for $2.5 \mathrm{~h}$ at room temperature, washed 10 min in PBS, rinsed with bidistilled water and finally dried. Then, all chips were incubated using $1 \mathrm{\mu g} / \mathrm{mL}$ Cy3-labelled antiCD81 IgG in PBS for $1 \mathrm{~h}$, washed $10 \mathrm{~min}$ in PBS, rinsed 
with bidistilled water and dried. Chips were finally analyzed using ExoView R100 to get postscan images.

\section{Results and discussion}

One of the significant strengths of a microarray analysis is the possibility to screen the affinity and reactivity of several probes towards a specific target simultaneously. Among diverse issues that might affect the performance of this technique, the most important is the coupling efficiency of the analyte to the surface. In particular, a good immobilization strategy that preserves the native conformation of probes, while providing optimal orientation is needed. In fact, the most common methods used for biomolecules immobilization, based both on non-specific adsorption and covalent binding, may suffer from binding activity loss and reduced probe conformational flexibility. The importance of probe orientation has been recently demonstrated by our group, immobilizing an azido modified peptide onto a slide coated with copolymers bearing alkyne functionalities. The azido modified peptide showed a higher fluorescence signal respect to the non-modified peptide, suggesting the importance of a proper probe orientation[16]. In addition, Gori and collaborators have demonstrated the favorable role of correct bioprobe orientation in discriminating seronegative from infected individuals, generating more-reliable and more-reproducible data[4]. Such orientation, obtained by chemoselective and biorthogonal click reactions, obviously necessitates the probe modification with click-compatible functionalities (for example alkyne or azide groups). This, consequently, forces the use of a surface coating bearing matching moieties, thus hindering the direct comparison of different species analyzed on the same support. As a result, probes must be immobilized on different slides and analyzed in different moments. Such a procedure, however, can be a source of systematic or random errors, leading to imprecise analyses. The possibility of immobilizing different chemical species on the same support (e.g., protein, oriented peptides, and DNA) represents a convenient method to overcome this issue, besides offering a drastic shortage in time, reagents and costs. However, this strategy requires the presence of distinct functionalities on the same surface. Over the last twenty years, our group has introduced several polymeric coatings to form a stable and hydrophilic nanometric film on various materials (glass, silicon, gold, thermoplastics) [17]. The amphiphilic behavior of $N, N$-dimethylacrylamide (DMA), which constitutes the polymer backbone, allows the binding to the substrate by a combination of hydrogen bonds, Van der Waals and hydrophobic interactions. Additionally, a silane 
monomer, in particular, 3-(trimethoxylsilyl)propyl methacrylate (MAPS) is incorporated to strengthen the bonding of the polymer to the surface through the formation of covalent bonds with hydroxyl or silanol groups. Finally, a third monomer confers chemical reactivity to the copolymer, particularly, $\mathrm{N}$-acryloyloxysuccinimide (NAS). We have recently exploited the high reactivity of NAS towards nucleophiles (such as amine) to generate, by post-polymerization modification, a family of polymers bearing functional groups that allow probe coupling through click chemistry reactions[18]. In this work, we present an easy and straightforward method to immobilize different probes (specifically proteins and oriented peptides) on the same substrate, exploiting NAS and azide groups' co-presence. In particular, the parent polymer bearing NAS functionalities is modified only in part using 3-azido-1-propylamine. The resulting polymer maintains the same characteristics of hydrophilicity and self-adsorption of the parent polymer, which has been extensively characterized in previous works[19][20]. The obtained polymers were extensively characterized using NMR to confirm the degree of NAS substitution and, similarly, their behavior in binding simultaneously protein, peptide and DNA probes was tested.

\subsection{NMR characterization}

The NMR spectra of the modified copolymers (Copoly Mix 2 and Copoly Azide 10\%) were compared with that of the parent polymer (MCP4) to confirm the modification of the NAS moieties (Figure 4a and $\mathrm{b}$ ). The red and green rectangles in Figure $4 \mathrm{a}$ and $\mathrm{b}$ highlight the spectral region of the ${ }^{13} \mathrm{C}$ NMR spectrum showing the key differences between the copolymers. The most representative signals are belonging to the carbonyl region ranging from 176 to 168 ppm (Figure 4a). In this region, the three signals belonging to the NAS carbonyls of MCP4 occurring at 173 ppm (peak 1), 171 ppm (peak 2) and 170 ppm (peak 3) (Figure 4a; red rectangles) shows a decreased value of the integral in Copoly Mix 2 and totally disappeared in Copoly Azide 10\% spectrum. In fact, defining that the integral value of peak at 174ppm, (most likely due to the backbone carbonyls) to 100, the values of the integral of peak 1, 2 and 3 resulted to be 28, 17 and 41 respectively for MCP4; the same peaks for Copoly Mix 2 (Figure 4a) show a value of 19,9 and 23 respectively, which is the half of the values of the same peak of the parent polymer. Finally, the same peaks almost disappear in the spectrum of Copoly Azide $10 \%$ with integral values of 5, 2 and 6 . This result suggests that a partial substitution of succinimide moieties with azide groups took part in Copoly Mix 2 while it was completed in Copoly Azide 10\%. 
Similarly, the aliphatic area of the ${ }^{13} \mathrm{C}$ NMR spectrum (Figure $4 \mathrm{~b}$ ), shows the presence of a broad signal in the Copoly Mix 2 (Figure 4b) at 48.5 ppm (green rectangle), corresponding to the signal of the carbon in alpha position respect to the azide group. This peak increases in the spectrum of Copoly Azide 10\% (Figure 4b), and it is totally absent in the spectrum of the parent polymer MCP4.
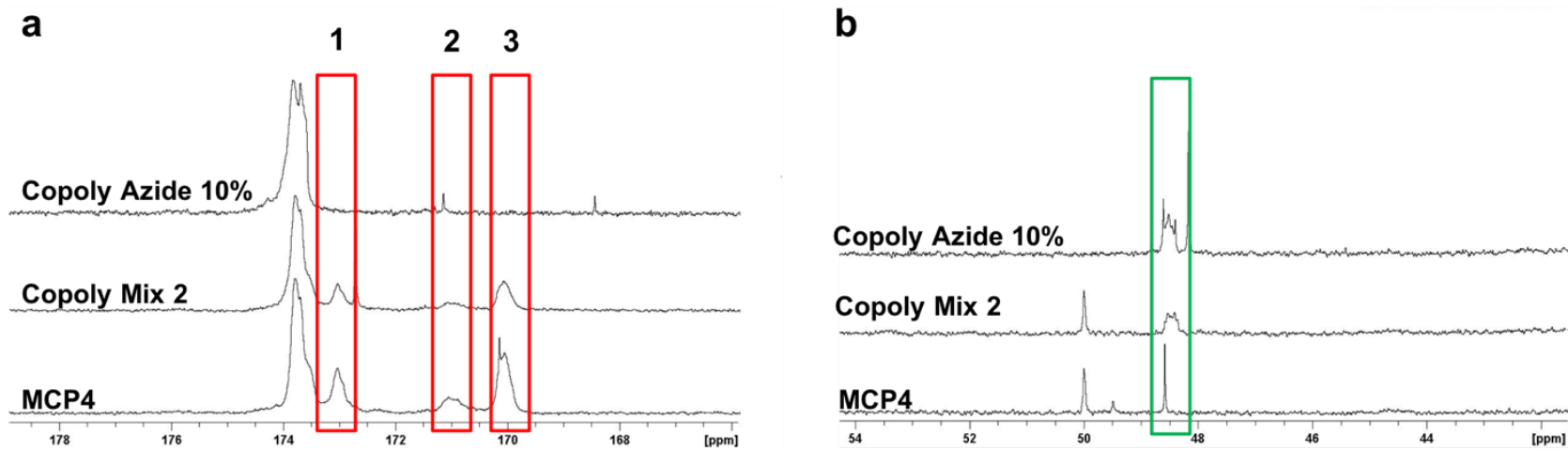

Figure 4a) Carbonyl region: in the spectrum of MCP4 it is possible to observe the presence of three signals (red rectangles) corresponding to the carbonyls of the succinimide group. The integrals of the signals are calculated as follows: 1=28, 2=17, 3=4. The same peaks, in the spectrum of Copoly Mix 2, have halved values: 1=19, 2=9, 3=23 and almost disappear in Copoly Azide 10\% in which all the succinimide moieties are modified with azido groups: 1=5, 2=2, 3=6. b) Aliphatic region: in the green rectangle the signal of the carbon in alpha position respect to the azide group is highlighted. This signal is present in Copoly Azide 10\% and partially in Copoly Mix 2 while is totally absent in MCP4, which does not contain any azide group.

\subsection{Surface functional test: DNA binding}

To test the degree of NAS substitution and the possibility of binding to the same surface two different functionalities, both amino and DBCO modified oligonucleotides, were spotted on silicon oxide chips modified with MCP4, Copoly Azide 10\%, Copoly Mix 2 and Copoly Mix 1. Oligonucleotides were spotted at $1,10,25$, and $50 \mu \mathrm{M}$ to test the binding capacity of each coated surface. After probe immobilization, each chip was incubated with the complementary DNA strand, fluorescently labeled to quantify the signal through a confocal laser scanner. 


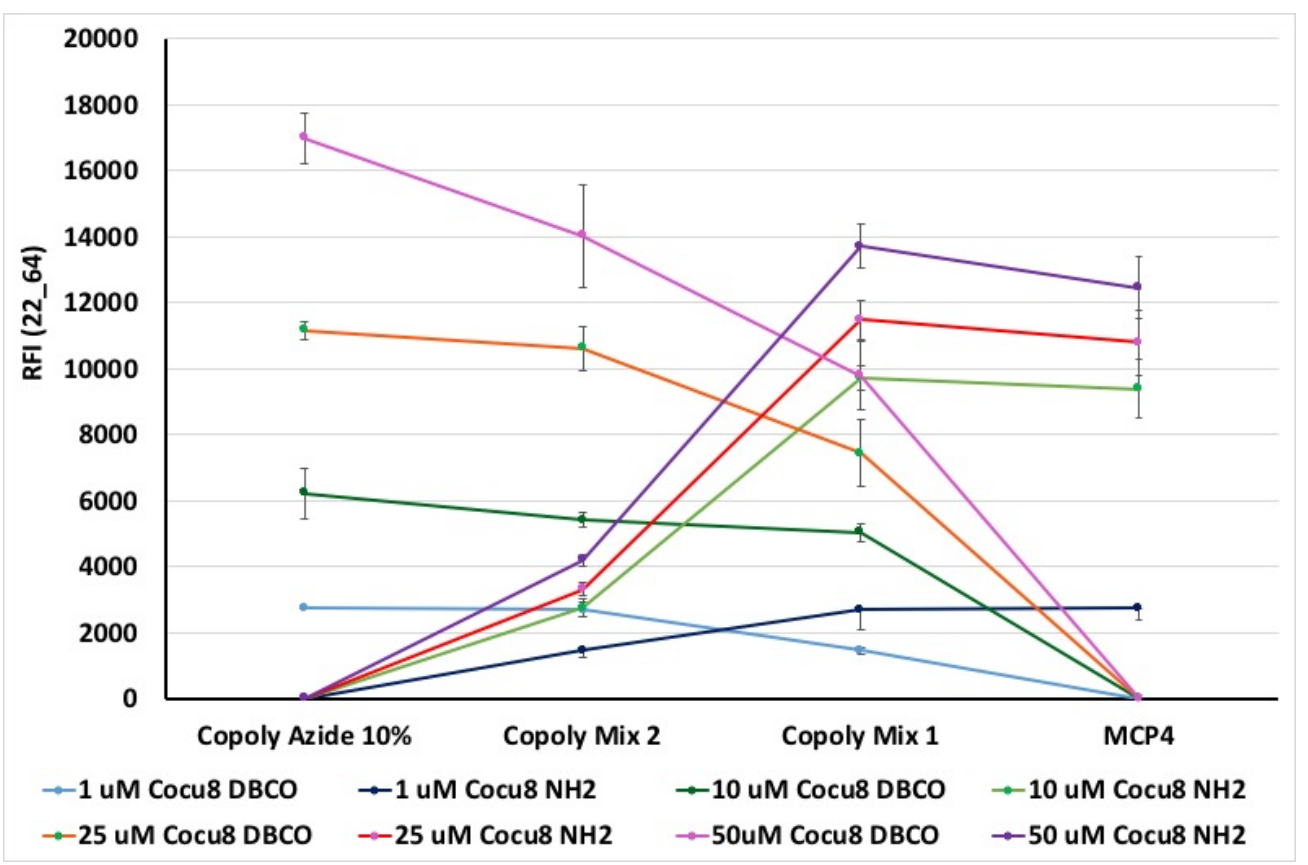

Figure 5 Fluorescence signals obtained after hybridization with Cy3 labelled oligonucleotide of an amino or DBCO modified single stranded DNA immobilized on MCP4, Copoly Azide 10\%, Copoly Mix 2 and Copoly Mix 1 coated chips. Single stranded oligonucleotides were spotted at increasing concentrations. Images were scanner setting the laser power at 22\% and the PMT gain at 64 .

Figure 5 shows the trend of fluorescence signals obtained after the hybridization of the probes (immobilized on the four coated surfaces) with the complementary Cy3 labelled oligonucleotide. The fluorescence signal on MCP4 reaches a plateau for a concentration of the immobilized aminomodified probe $\left(\mathrm{COCU} 8 \mathrm{NH}_{2}\right.$ ) higher than $10 \mu \mathrm{M}$, while the signal of Copoly Azide $10 \%$ shows a linear increase up to $50 \mu \mathrm{M}$, suggesting a difference in the probe density that can be reached on the various substrates. The same trend is reflected by the partially modified copolymers Copoly Mix 1 and Copoly Mix 2. Copoly Mix 1 has a low concentration of azide groups and shows a behavior more similar to MCP4; on the other hand, Copoly Mix 2 trend is comparable to the surface wholly modified with azide moieties.

The absence of COCU8 DBCO signals on MCP4 (and similarly of COCU8 $\mathrm{NH}_{2}$ on Copoly Azide 10\%; see also Figure $2 \mathrm{~b}$ ) indicates the specificity of the binding mediated by nucleophile substitution in case of aminated probe on MCP4 and click chemistry reaction on Copoly Azide 10\% surfaces. Furthermore, the absence of COCU8 $\mathrm{NH}_{2}$ signal on Copoly Azide $10 \%$ also demonstrates the postpolymerization modification of NAS moieties into azide groups is quantitative.

\subsection{Protein and peptide co-immobilization}

To test the possibility of immobilizing simultaneously on the same slide, a protein and an alkynemodified peptide, four silicon chips were functionalized with MCP4, Copoly Mix 1, Copoly Mix 2, and Copoly Azide $10 \%$. As a proof of concept, on each slide, a solution of $1 \mathrm{mg} / \mathrm{mL}$ Rabbit IgG in PBS was 
immobilized. Similarly, a $100 \mu \mathrm{M}$ solution of the E01 peptide was spotted, both in the presence or in the absence of $\mathrm{CuSO}_{4}$ and ascorbic acid. E01, used as a proof of concept, is a peptide able to mimic the antigenic performance of Dengue envelope protein $(E)$, which forms the outermost layer of the virion particle. A recent paper demonstrated that its immunodiagnostic performance is compared with that of the full protein E, also in discriminating Zika infected individuals from healthy controls [21]. Chips were then incubated with $0.1 \mathrm{mg} / \mathrm{mL}$ solution of a rabbit antibody directed against the E01 epitope in PBS and finally with a Cy3 labelled goat anti-Rabbit lgG $1 \mathrm{ug} / \mathrm{mL}$ in PBS for fluorescence detection.

a

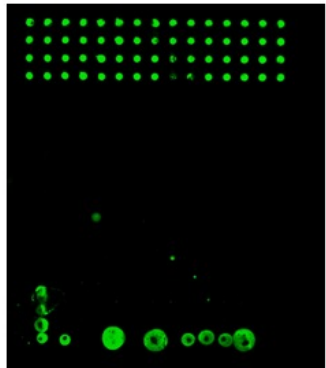

MCP4

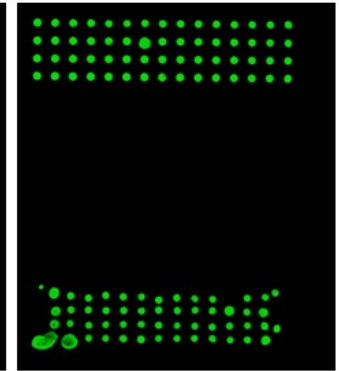

Copoly Mix 1

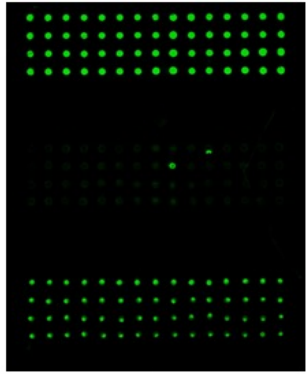

Copoly Mix 2

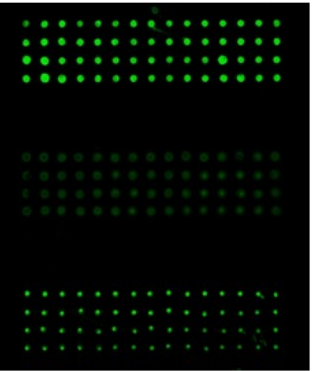

Copoly Azide $10 \%$
Rabbit IgG

Peptide E01 no

click

Peptide E01 click

b

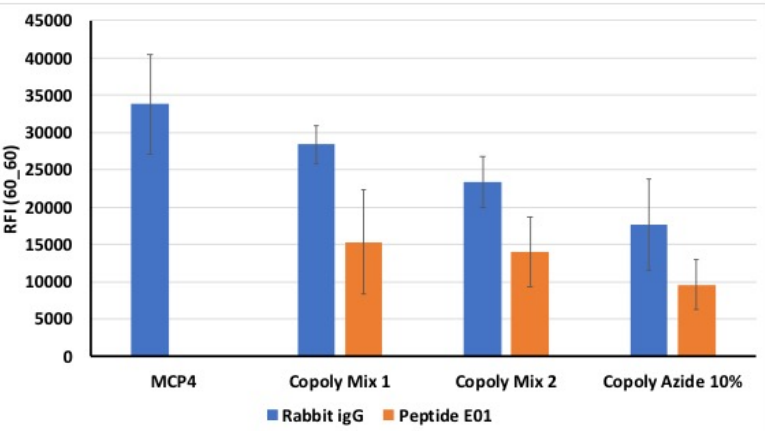

Figure 6 a) fluorescence images obtained after hybridization with Cy3-labelled goat anti Rabbit IgG on MCP4, Copoly Azide 10\%, Copoly Mix 2 and Copoly Mix 1 coated chip where both rabbit IgG and peptide E01 were immobilized; b) fluorescence intensity signals obtained setting the laser power at $60 \%$ and PMT gain at $60 \%$.

Figure 6 shows the fluorescence images (Figure 6 a) obtained after incubation with Cy3-labelled goat anti Rabbit IgG and the relative signal intensity (Figure 6 b). A fluorescence signal for the Rabbit IgG is clearly visible on each slide, even if the signal on MCP4 is remarkably higher than the signal obtained on Copoly Azide 10\% (Figure 6 b). This result suggests that a non-specific binding due to hydrophobic, van der Waals, and electrostatic interactions is present on each surface. However, the binding on MCP4 is mainly covalent due to the reaction between NAS and amino groups on the protein.

On the contrary, the fluorescence signal of the peptide E01 is detectable only on surfaces bearing azide groups, meaning that the 1,3-dipolar cycloaddiction click reaction mediates the binding between the alkyne group of the peptide and the azide moieity on the chip surface. This is further confirmed by the absence of the fluorescence signal of the peptide spotted without the click 
reaction mix catalysts (Figure 6 a, Peptide E01 no click). A similar experiment was conducted as a negative control, skipping the incubation with anti E01 antibody (data not shown). In this case, no fluorescence signal was detected for peptide E01, indicating that the secondary fluorescent antibody binding is specific.

Interestingly, increasing the percentage of azide moieties does not significantly improve the signal intensity; however, as can be noticed from the fluorescence images, the spot morphology ameliorates. This further endorses the theory that, depending on the probe that has to be immobilized, the density of functional groups is not the only parameter to take into account. Still, the degree of hydrophilicity should be equally considered to achieve a proper probe immobilization[22].

\subsection{Extracellular vesicles capture and characterization}

Having demonstrated the possibility of immobilizing different probes on the same surface by exploiting simultaneously amine coupling and copper catalyzed azide-alkyne cycloaddition (CuAAC), we have chosen Copoly Mix 2 for the analysis of extracellular vesicles (EVs), as it showed the best performance and spot morphology in the previous experiments.

EVs are membrane bound vesicles released from cells into bodily fluids that, upon internalization into recipient cells, carry information in the shape of nucleic acids, lipids and proteins [23]. EVs are involved in a plethora of biological processes, in physiological as well as pathological conditions, and are eligible as biomarkers for liquid biopsy [24][25]. For this reason, the analysis of EVs can potentially become an essential tool not only for the diagnosis of multiple pathologies but also to monitor their progression over time.

An important example is given by neurodegenerative disorders, whose diagnosis is often limited by the inaccessibility of biomarkers. Neural EVs, being able to cross the blood brain barrier, can be found in plasma and are thus eligible tools for the diagnosis at an early stage of these kind of diseases [26].

In the last few years Nanoview Biosciences (Boston, MA, USA) has commercialized ExoView R100, an instrument that, combining label free interferometry and fluorescence detection on an antibody microarray platform, provides EVs counting and phenotyping down to single particle level [27][28]. Exploiting this technology, we have recently developed both antibody and peptide microarrays to perform EVs characterization successfully $[14,29]$. Previously, these different approaches could not be directly compared on the same surface since, as already mentioned, to reach an optimal orientation, peptides require a surface chemistry different from that needed by native antibodies, thus increasing variability and limiting reproducibility. As stated before, the correct orientation of 
the immobilized probe, especially of a peptide is fundamental to maximize binding efficiency and achieve consistent and reproducible data, enhancing the statistical significance of the analytical assay performance[4].

Within this work, we exploited Copoly Mix 2 to generate a microarray platform that bears both azide and NAS functionalities, able to bind simultaneously alkyne-modified peptides and native anitibodies (see Section 2.6.1). Using this strategy we immobilized two antibodies directed against two members of tetraspanin family, which are exposed on the membrane of EVs, namely CD9 and CD63, and a membrane-sensing peptide that is able to bind small EVs, called BP. In particular, BP is a short peptide (sequence: RPPGFSPFR) derived from bradykinin that was reported to bind in solution to highly curved lipid nanovesicles. BP peptide is a general binder for the highly curved membranes of small EVs ( $100-150 \mathrm{~nm})$; its recognition mechanism is not mediated by the proteins expressed on the surface of EVs (like antibodies do) and has been demonstrated to possess higher EVs binding capacity and lower limit of detection when compared to antibodies [14].

Similarly, two negative controls were also immobilized on the same chip. In particular, a rabbit IgG was immobilized together with a peptide bearing opposite charge of BP since glutamic acid residues were substituted with arginine residues (BPn) [14].

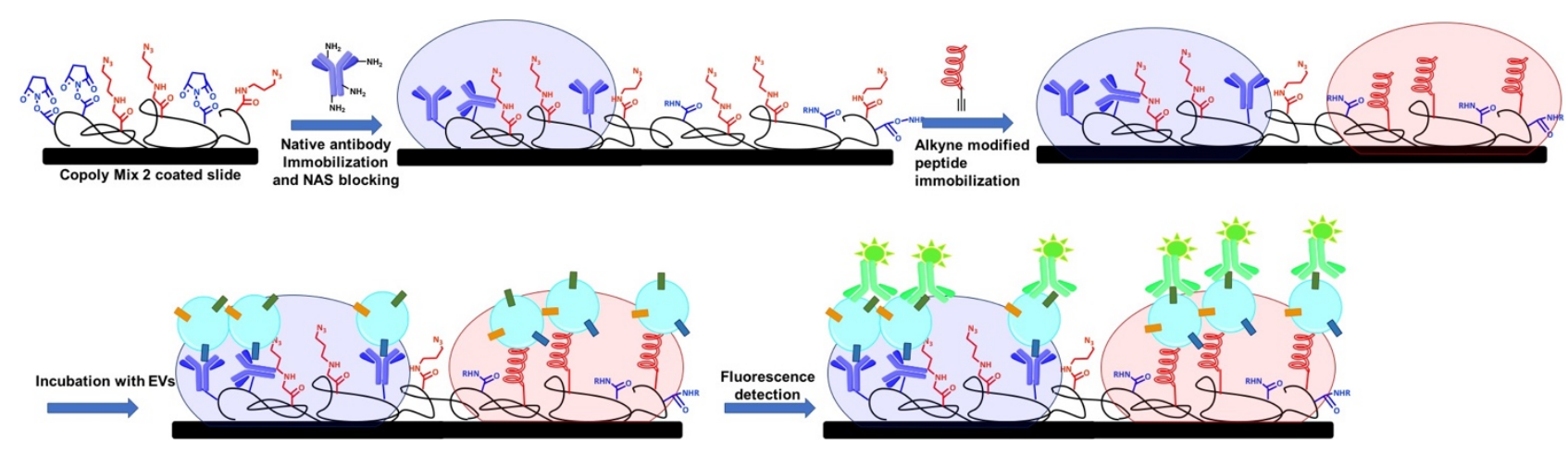

Figure 7 Schematic representation of the EVs assay: on a chip coated with Copoly Mix 2, bearing both azide and NAS functionalities antibodies anti CD9 and anti CD63 were immobilized by spotting. After NAS blocking, an alkyne modified peptide (BP) was immobilized as well. The chip was then incubated with EVs (obtained by ultracentrifugation of HEK-293 cell culture supernatant) and finally incubated with a Cy3-labelled anti CD81 for fluorescent detection with ExoView ${ }^{T M} R 100$.

EVs were isolated from HEK-293 cell culture supernatant through ultracentrifugation and NTA assessed their concentration. The vesicle-containing sample was diluted to a final concentration of $1 * 10^{9}$ particles/mL using PBS, and the so-obtained solution was used to incubate microarray chips as described in Section 2.6.2 (see Figure 7). As a negative control, a group of chips was incubated with PBS. Both groups were then incubated with a Cy3-labelled antibody directed against a different tetraspanin (CD81) present on the vesicles membrane. Finally, label free interferometry as well as fluorescence detection of nanoparticles was performed using ExoView ${ }^{\mathrm{TM}}$ R100, a platform that 
provides digital count, size and phenotyping of individual and low-refractive index nanoparticles (50-200 $\mathrm{nm}$ in diameter) [28].

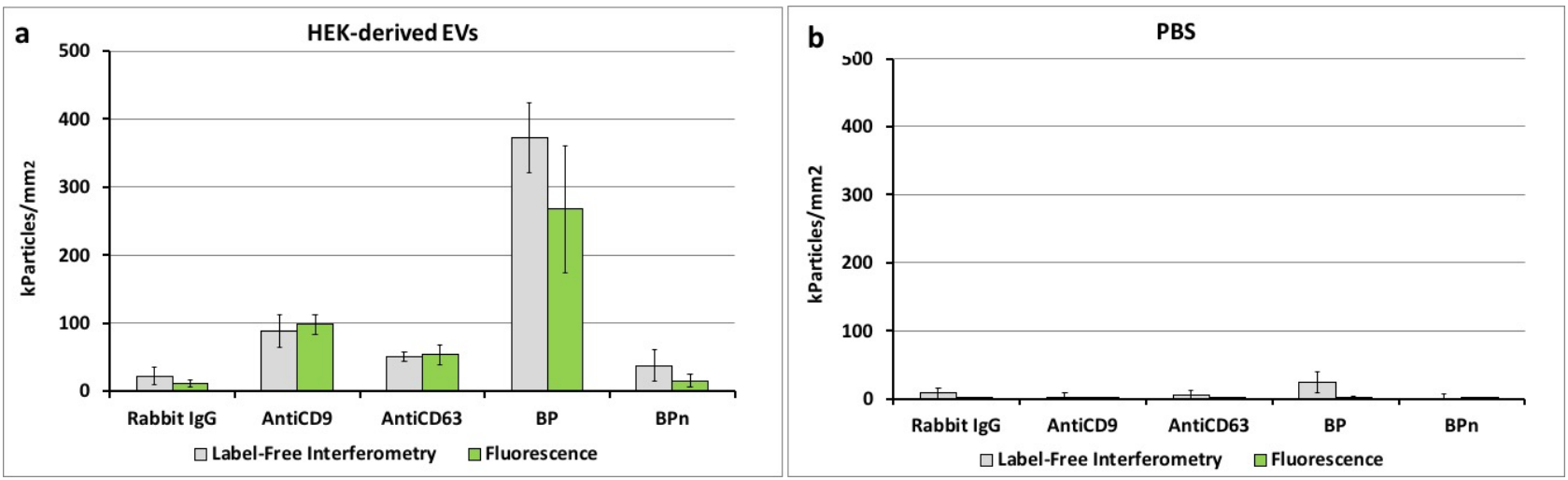

Figure 8 a) Label free and fluorescence signals obtained after incubation of a microarray chip, coated with Copoly Mix 2 and spotted with CD9 and CD63, with HEK-293 separated EVs. Fluorescence detection is obtained by a second incubation with a Cy3-labelled anti CD81 antibody. Specific signals are detected for antiCD9 anti CD63, meaning EVs are captured by these antibodies, and similarly, EVs are captured also by peptide $B P$; b) negative control experiments obtained by incubating chip with PBS instead of EVs. No signals are detected demonstrating the specificity of the assay.

Figure 8 demonstrates that the direct comparison between antibodies and peptide could be performed on the same chip. In fact, as shown in Figure 8a, clear, specific signals (both fluorescence and label free) are detected for antiCD9 anti CD63, meaning EVs are captured by these antibodies through recognition of the correspondent membrane proteins. Similarly, EVs are captured also by peptide BP. As expected, the peptide BP signal is higher than that of the antibodies, since peptide BP binds small EVs independently from their protein composition [14]. At the same time, the antibody-mediated interaction captures only a specific subpopulation of EVs. In all cases, the signals are specific, since both negative controls (rabbit IgG and peptide BPn) show negligible signals. The EVs immobilization density of both antibodies and peptide is 50-100 and $300 \mathrm{kParticles} / \mathrm{mm} 2$ respectively, consistent with previous findings [14,29]. Furthermore, the system displays a good correspondence between label free and fluorescence detection (Figure 8a) confirming that interferometric signals, are actually referred to extracellular vesicles and not due to non-specific binding (e.g. protein aggregates). On the contrary, chips incubated only with PBS and no EVs (Figure $8 b)$ show no detectable signal. Using the herein described surface chemistry it is possible to combine on the same analytical surface both the overall concentration of small EVs (signal on BP peptide which binds to EVs independently from their membrane protein composition and directly relates to vesicles' concentration) and the membrane protein phenotyping by quantification of the relative abundance of specific subpopulations (in this example CD9+ and CD63+ vesicles, captured using antibodies). 


\section{Conclusion}

We have introduced a new family of copolymers which bear two functionalities (azide and active ester) that allows the simultaneous immobilization of different probes by exploiting different chemical reactivities. In particular, it was demonstrated that probes bearing amino groups and triple bonds (alkyne or DBCO groups) can be bound on the same chip, maintaining their activity towards their targets through proper surface orientation. This strategy consents to compare directly the performance of different bioprobes in capturing targets, thus reducing the number of experiments and possible errors. In particular, these copolymers' use as surface coating, allows the direct comparison of antibodies and peptides in capturing EVs. Potentially, the use of this kind of surface chemistry, would enable discriminating simultaneously the percentage abundance of different subpopulations of EVs into a specific sample.

\section{Acknowledgements}

We acknowledge funding by European Union's Horizon 2020 research and innovation programme under grant agreement $n^{\circ} 766466$ (project INDEX), and by Regione Lombardia, project READY (Regional Network for developing diagnostic methods in rapid response to emerging epidemics and bio-emergencies) ID 229472.

\section{References}

[1] L. Wu, P. Mickey Williams, W.H. Koch, Clinical applications of microarray-based diagnostic tests, Biotechniques. 39 (2005) S557-S582. doi:10.2144/000112046.

[2] D. Chandler, L. Bryant, S. Griesemer, R. Gu, C. Knickerbocker, A. Kukhtin, J. Parker, C. Zimmerman, K. George, C. Cooney, Integrated Amplification Microarrays for Infectious Disease Diagnostics, Microarrays. 1 (2012) 107-124. doi:10.3390/microarrays1030107.

[3] E.F. Petricoin, J.L. Hackett, L.J. Lesko, R.K. Puri, S.I. Gutman, K. Chumakov, J. Woodcock, D.W. Feigal, K.C. Zoon, F.D. Sistare, Medical applications of microarray technologies: A regulatory science perspective, Nat. Genet. 32 (2002) 474-479. doi:10.1038/ng1029.

[4] A. Gori, L. Sola, P. Gagni, G. Bruni, M. Liprino, C. Peri, G. Colombo, M. Cretich, M. Chiari, Screening Complex Biological Samples with Peptide Microarrays: The Favorable Impact of Probe Orientation via Chemoselective Immobilization Strategies on Clickable Polymeric 
Coatings, Bioconjug. Chem. 27 (2016). doi:10.1021/acs.bioconjchem.6b00426.

[5] S. Noel, C. Fortier, F. Murschel, A. Belzil, G. Gaudet, M. Jolicoeur, G. De Crescenzo, Coimmobilization of adhesive peptides and VEGF within a dextran-based coating for vascular applications, Acta Biomater. 37 (2016) 69-82. doi:10.1016/j.actbio.2016.03.043.

[6] S. Pernagallo, J.J. Diaz-Mochon, Polymer Microarrays for Cellular High-Content Screening, in: Methods Mol. Biol., 2011: pp. 171-180. doi:10.1007/978-1-61737-970-3_14.

[7] A.L. Hook, C.-Y. Chang, J. Yang, D.J. Scurr, R. Langer, D.G. Anderson, S. Atkinson, P. Williams, M.C. Davies, M.R. Alexander, Polymer microarrays for high throughput discovery of biomaterials., J. Vis. Exp. (2012) e3636. doi:10.3791/3636.

[8] S. Rigo, G. Gunkel-Grabole, W. Meier, C.G. Palivan, Surfaces with Dual Functionality through Specific Coimmobilization of Self-Assembled Polymeric Nanostructures, (2018). doi:10.1021/acs.langmuir.8b02812.

[9] X. Deng, J. Lahann, A Generic Strategy for Co-Presentation of Heparin-Binding Growth Factors Based on CVD Polymerization, Macromol. Rapid Commun. 33 (2012) 1459-1465. doi:10.1002/marc.201200343.

[10] L. Sola, F. Damin, P. Gagni, R. Consonni, M. Chiari, Synthesis of clickable coating polymers by postpolymerization modification: Applications in microarray technology, Langmuir. 32 (2016) 10284-10295. doi:10.1021/acs.langmuir.6b02816.

[11] M. Mammen, G. Dahmann, G.M. Whitesides, Effective Inhibitors of Hemagglutination by Influenza Virus Synthesized from Polymers Having Active Ester Groups. Insight into Mechanism of Inhibition, J. Med. Chem. 38 (1995) 4179-4190. doi:10.1021/jm00021a007.

[12] F. Landi, C.M. Johansson, D.J. Campopiano, A.N. Hulme, Synthesis and application of a new cleavable linker for "click"-based affinity chromatography., Org. Biomol. Chem. 8 (2010) 5659. doi:10.1039/b916693a.

[13] G. Bergamaschi, E.M.A. Fassi, A. Romanato, I. D’Annessa, M.T. Odinolfi, D. Brambilla, F. Damin, M. Chiari, A. Gori, G. Colombo, M. Cretich, Computational Analysis of Dengue Virus Envelope Protein (E) Reveals an Epitope with Flavivirus Immunodiagnostic Potential in Peptide Microarrays, Int. J. Mol. Sci. 20 (2019) 1921. doi:10.3390/ijms20081921.

[14] A. Gori, A. Romanato, B. Greta, A. Strada, P. Gagni, R. Frigerio, D. Brambilla, R. Vago, S. Galbiati, S. Picciolini, M. Bedoni, G.G. Daaboul, M. Chiari, M. Cretich, Membrane-binding peptides for extracellular vesicles on-chip analysis, J. Extracell. Vesicles. 9 (2020) 1751428. doi:10.1080/20013078.2020.1751428. 
[15] L. Sola, F. Damin, P. Gagni, R. Consonni, M. Chiari, Synthesis of clickable coating polymers by postpolymerization modification: Applications in microarray technology, Langmuir. 32 (2016) 10284-10295. doi:10.1021/acs.langmuir.6b02816.

[16] L. Sola, A. Gori, M. Cretich, C. Finetti, C. Zilio, M. Chiari, Clickable Polymeric Coating for Oriented Peptide Immobilization, in: 2016: pp. 167-182. doi:10.1007/978-1-4939-30371_13.

[17] C. Zilio, L. Sola, F. Damin, L. Faggioni, M. Chiari, Universal hydrophilic coating of thermoplastic polymers currently used in microfluidics, Biomed. Microdevices. 16 (2014) 107-114. doi:10.1007/s10544-013-9810-8.

[18] L. Sola, F. Damin, P. Gagni, R. Consonni, M. Chiari, Synthesis of clickable coating polymers by post-polymerization modification: application in microarray technology, Langmuir. 32 (2016) 10284-10295.

[19] L. Sola, M. Chiari, Modulation of electroosmotic flow in capillary electrophoresis using functional polymer coatings, J. Chromatogr. A. 1270 (2012) 324-329. doi:10.1016/j.chroma.2012.10.039.

[20] R. Suriano, M. Levi, G. Pirri, F. Damin, M. Chiari, S. Turri, Surface behavior and molecular recognition in DNA microarrays from $\mathrm{N}, \mathrm{N}$-dimethylacrylamide terpolymers with activated esters as linking groups., Macromol. Biosci. 6 (2006) 719-29. doi:10.1002/mabi.200600088.

[21] G. Bergamaschi, E.M.A. Fassi, A. Romanato, I. D’Annessa, M.T. Odinolfi, D. Brambilla, F. Damin, M. Chiari, A. Gori, G. Colombo, M. Cretich, Computational analysis of dengue virus envelope protein $(E)$ reveals an epitope with flavivirus immunodiagnostic potential in peptide microarrays, Int. J. Mol. Sci. 20 (2019) 1-13. doi:10.3390/ijms20081921.

[22] L. Sola, F. Damin, M. Cretich, M. Chiari, Novel polymeric coatings with tailored hydrophobicity to control spot size and morphology in DNA microarray, Sensors Actuators, B Chem. 231 (2016) 412-422. doi:10.1016/j.snb.2016.03.049.

[23] M.P. Zaborowski, L. Balaj, X.O. Breakefield, C.P. Lai, Extracellular Vesicles: Composition, Biological Relevance, and Methods of Study, Bioscience. 65 (2015). doi:10.1093/biosci/biv084.

[24] M. Borghesan, J. Fafián-Labora, O. Eleftheriadou, P. Carpintero-Fernández, M. Paez-Ribes, G. Vizcay-Barrena, A. Swisa, D. Kolodkin-Gal, P. Ximénez-Embún, R. Lowe, B. Martín-Martín, H. Peinado, J. Muñoz, R.A. Fleck, Y. Dor, I. Ben-Porath, A. Vossenkamper, D. Muñoz-Espin, A. O'Loghlen, Small Extracellular Vesicles Are Key Regulators of Non-cell Autonomous 
Intercellular Communication in Senescence via the Interferon Protein IFITM3, Cell Rep. 27 (2019) 3956-3971.e6. doi:10.1016/j.celrep.2019.05.095.

[25] M. Harmati, E. Gyukity-Sebestyen, G. Dobra, L. Janovak, I. Dekany, O. Saydam, E. HunyadiGulyas, I. Nagy, A. Farkas, T. Pankotai, Z. Ujfaludi, P. Horvath, F. Piccinini, M. Kovacs, T. Biro, K. Buzas, Small extracellular vesicles convey the stress-induced adaptive responses of melanoma cells, Sci. Rep. 9 (2019) 1-19. doi:10.1038/s41598-019-51778-6.

[26] A.F. Hill, Extracellular Vesicles and Neurodegenerative Diseases, J. Neurosci. 39 (2019) 9269-9273. doi:10.1523/JNEUROSCI.0147-18.2019.

[27] Complete Exosome Characterization, ExoView R100, (n.d.).

[28] G.G. Daaboul, P. Gagni, L. Benussi, P. Bettotti, M. Ciani, M. Cretich, D.S. Freedman, R. Ghidoni, A.Y. Ozkumur, C. Piotto, D. Prosperi, B. Santini, M.S. Ünlü, M. Chiari, Digital Detection of Exosomes by Interferometric Imaging, Sci. Rep. 6 (2016) 1-10. doi:10.1038/srep37246.

[29] D. Brambilla, L. Sola, M. Chiari, Advantageous antibody microarray fabrication through DNAdirected immobilization: A step toward use of extracellular vesicles in diagnostics, Talanta. 222 (2021) 121542. doi:10.1016/j.talanta.2020.121542. 